\title{
The Translation Methods of Cosmetic Brand Name
}

\author{
Yu Zhang \\ Foreign Language Department \\ The Engineering and Technical College of Chengdu University of Technology \\ Leshan, China
}

\begin{abstract}
With the rapid development of economy, commercial activity grows steadily, and people are pursuing for more luxurious, higher quality of life. As to cosmetic enterprise, what is important to the sellers is how to make their products more fascinating to attract more eyeballs. With a good brand name translation, the brand can make success among a variety of cosmetics in the violent market competition. So the foreign sellers intend to expend much more thoughts in building a brand image as they access to Chinese market. And domestic cosmetic companies focus on stepping into the foreign market using a good brand name translation. As a kind of special consumer goods, the makeup cosmetics attract a lot of attention. There are more and more multiple types of cosmetic from home and abroad, which sell well in the market. Good sale volume is closely related to the suitable translation of the brand names. Therefore, this paper analyzes the cosmetic brand name translation on the basis of disciplines of brand translation and stylistic features, and combines it with rules of word selection and lexical characteristics.
\end{abstract}

Keywords — brand name; cosmetic; translation methods

\section{INTRODUCTION}

The development of economy leads to the popularity of cosmetics, and it has become the third most important consumer product in China. As China's accession to the WTO, a large number of foreign famous cosmetics swarm into Chinese market. And Chinese traditional brands are exploring their own way to internationalization. Therefore, cosmetic brand translation, which is one important branch of pragmatic translation, is becoming a key way in the development of cosmetics. It is not only a marketing strategy, but also a collision of traditional culture and western civilization. [1]

As we know, most consumers of cosmetics are female, so the key point of cosmetic brand translation should make consumer feel beautiful. The mission of translators is giving the brand an associative, suggestive and beautiful translation. Therefore, the good volume and the fitting translation are closely related.

Such as the brand "CPB", which aims at high level market, has amazing sales volume, the Chinese translation is “Ji Fu Zhi Yue” (肌肤之钥), “Ji Fu” means the skin, “Yue” has two pronunciations, but they all means the key, the one is the key we use to open the door, the other one means the key point. It undoubtedly deepens inviolable cultural implication of brand. So, when the domestic consumers see the brand translation, they may associate a gold key which can open the door of beauty. In this thesis, the author analyzes cosmetics brand translation due to two aspects of principles, linguistic advantages and cultural identity. Moreover, the thesis proposed four translation methods of cosmetic brand name translation.

\section{OVERVIEW OF BRAND NAME}

In this chapter, the basic features of brand name will be introduced for its concentration and identification, and the functions of brand name will also be introduced on its value embodiment and consumer appealing.

\section{A. The Features of the Brand Names}

In this section, the basic features of the brand name for its concentration and identification will be introduced

1) The Concentration of One Company: Cosmetic translation refers to the translation of all relevant text and discourse, including brand name, brand add, product characteristics, instructions, and so on. This essay focuses on the cosmetic brand name translation, for the reason that brand name is the concentration of one company or one product. As the open up of the market, a large number of foreign commodities are flooding into the Chinese market. Trademark is the symbol of the commodity, but also the symbol of the enterprise. It can not only reflect the image of the enterprise, but also display the product's service and reputation. With the expansion of trademark communication enterprises can gain market recognition, and reputation. [2] Brand-name goods once determined can bring huge profits to enterprises. The quality of raw materials or ingredients, the same as similar products, brand-name products can be several times higher than the average price of the brand. The brand can distinguish the brand's products or services and other goods, highlight their own characteristics, to encourage consumers to buy directional. A strong brand, is a widely used, and can withstand the test of time brand. An enterprise can spread information like its industry, various functions, or its company culture. When we met with a variety of products, we notice its brand name at the first sight. As a result, how to use a brand name to transmit one company is of vital importance.

2) Identification Expediently: Cosmetic brand translation has a number of utilities. Information 
dissemination is one of the most important utility. That is to say, cosmetic brand name can describe the products, thus to communicate and resonate with the customers. We cannot identify what kind of customers can be attracted. Otherwise, we can put the brand name identification expediently. Easy to accept is a prerequisite for the commercial benefits of cosmetics. The principle of expediently identification is from the cross cultural communication. [3] Culture is a complex unit which includes knowledge, belief, art, morals, law, custom, and people as members of society and gets all the abilities and habits.

The trademark translation is not only a process of the transformation of two different languages; more is to consider the cross cultural communication. For truly successful translating, familiar with the two different cultures is even more important than master two languages. [4] One brand who is easy to identify is the one who can make customers impressed at the first sight. If one brand is long, copious and boring, its market share is doomed to be grabbed by other similar brand. Otherwise, one concise, clear and funny brand can draw people's attention and make more profit, more sales volume.

\section{B. The Functions of the Brand Name}

In this section, the functions of the brand name for value embodiment and consumer appealing will be introduced.

1) Value Embodiment: The essay put a big emphasis on value embodiment, which is the basis discipline of cosmetic brand translation. Easily identified and appealing is not enough for a brilliant cosmetic brand translation. According to the information requirements of functional equivalence, translation of brand names should be characterized as possible and with the information contained. People may not go into a brand which cannot be read its value by its brand name when they have a clear target to buy clothes or buy cosmetics. As a result, value embodiment is the basic rule of cosmetic translation. For example, people may be attracted by the name “Ke Ling Ke Li" (可伶可俐) when in their youth, because “Ling Li"(伶俐) in Chinese means clever and beauty. It is consistent with the original brand positioning, and reflect the value of product at the same time.

2) Consumer Appeal: The leading function of cosmetic brand name is consumer appeal. Cosmetics have a close relationship with beauty. The main target of using cosmetics is to beautify one's appearance. Therefore, cosmetic brand name itself should also be a form of beauty, have a strong sense of rhythm, can initiate people's better association, thus to appeal people's big appetite for buying. Brand name translation should be meaningful, but only meaningful cannot guarantee the success of trademark. It should also have aesthetic significance. [5] A creative and creative translation can be more attractive to consumers. Here is not just to attract consumers to stop and see, but let her see the name cannot help but produce a desire to buy. Consumers can through the brand name associated with the product use, efficacy, thus in itself does not need, also want to buy home for a stockpile. A good cosmetic brand translation can not only translate the benefit of using it, but also can draw people's attention in the sea of various brand names, in the accelerated tempo of life, and in the complex society. By the wonderful cosmetic names it translated from one language into other language, the cosmetics can convey its beautiful meaning and imposing image to their potential foreign customers.

\section{THE PRINCIPLES OF BRAND TRANSLATION}

\section{A. Abbreviations and Acronyms}

A good cosmetics brand must follow the principle of simplicity, so that readers can easily remember and understand the brand. For example, "Pure Mild" is translated as "Bo Mei" (泊美), with two short words which mean staying in the harbor of beauty in Chinese. Compared with original brand name, "Bo Mei" is easier to be remembered, and is good for the opening of domestic market. Similarly, "Yves Saint Laurent" (伊夫圣罗兰) uses its acronym "YSL" and translated as "Sheng Luo Lan" (圣罗兰), with three capital letters make people have a deepen impression about the brand.

Studies show that short and clear word can live long in man's brain than long and complex ones. So that, clear and intelligible brand name can leave a deep impression in customers' memory, thus they can be spread from mouth to mouth, and conveyed from one to others. [6] Moreover, there is a cognitive gap between different cultures. To be specific, there is a difference between a country's cosmetic promotion and another country's reader's cognitive context. A word in western culture may mean nothing in eastern culture, vice verse. Translators have to know deeply what customers want in the target market, and make it clear, because they know that people tend to use the lowest cognitive effort to achieve the most effective contextual effect.

\section{B. Innovative and Fantastic}

In brand name translation, translators tend to do their best to seek the most effective way to attract readers, in order to arouse the reader's desire to purchase. Brand name is the product of strong communication. The translation must have the same ultimate purpose with the original one, which is his implementation of cosmetics sales targets, and the investment into consumer's favor. The final destination of cosmetic brand name translation is to impress them, and to make the purchasing action.

There are a series of way to make brand name innovative and fantastic. On one hand, the use of independent word or phrase can enhance the attractiveness. As a result, new words may not only keep the original meaning, but also be rich in new ideas, which can produce funny and innovative atmosphere. This replacement makes people memorable. For example, "3 Concept Eyes" is translated as "3CE"; it does not conform to Chinese translation custom, but it also popular in young people. 
On the other hand, cosmetic is the product of times. The translation of cosmetic brand name should reflect the cultural atmosphere of the times. People may use the commendatory background in Chinese, who is on behalf of the beauty. So that it can be consistent with the public aesthetic demand, and fill the product with the art of language. [7]

This method is the way to make products closer to the people, closer to life, more vivid, and full of charisma. Such as "Shiseido" is translated "Zi Sheng Tang" (资生堂) in Chinese, "Zi Sheng" comes from the Chinese ancient book The Book of Changes, means new life, the creative translation bring a lot of consumers.

\section{Extension and Association}

Buy by its name tends to be the habit of purchasing nowadays. Cosmetic brand name translation is not a simple translation, it should be meaningful. People can produce extension and association by its name. Only by this, brand can enhance its sales volume and popularity. This kind of lexical character is well presented in Johnson's, a famous baby care brand. Johnson's is just a family name of the original founder of the brand. It is simple and meaningless. In Chinese translation, the brand is translated as "Qiang Sheng" (强生), which is similar to the pronunciation of "Johnson's". However, it has rich connotation, which can make people associate it to babies' health care and healthier growth. Similarly, "Revlon" is known as "Lu Hua Nong" (露 华浓) in China, not only for its famous advertisement The most unforgettable women in the world use "Revlon", but also for "Lu Hua Nong", which makes people associate with beautiful morning dew, just like women's exquisite and smooth skin. Literal translation can faithfully reproduce the original meaning, which makes it easier to be accepted by the vast number of Chinese consumers.

\section{Feminized Diction}

Target customer is the key in marketing strategy. Cosmetics are faced with the vast female customers. As a result, feminized diction will be a good choice when people are translating a cosmetic brand.

There is no doubt that a brand name with a beautiful and feminine meaning will help arouse emotions in the female consumer groups, and make them more likely to have a sense of beauty. Female consumers can sometimes be easily persuaded by logical theory. However, they are always be convinced by products' symbolic meaning. A good translation can convey feelings of sweet and happy to female customers. It can give women unlimited imagination. This kind of pleasure will help them have a good impression on the goods, and when they are choosing cosmetics, they will tend to the brand. Such as the "Estee Laude", it translated as "Ya Shi Lan Dai" (雅诗兰黛), this four Chinese words are typical female words which can make the person produces beautiful reverie.

In order to obtain a better sales performance, translators need to create a new trademark by translating, taking advantage of its freedom and flexibility, to arouse women's enjoy on the cosmetic. [8] This can be made success by the way of voice and meaning of the original brand. For women customers, they tend to be fond of gentle and sweet words. For example, the translation of purple perfume "Poison" as “Bai Ai Shen" (百爱神) is a good challenge for cosmetic brand translation. If "Poison" is translated to "Du Yao" (毒药) in Chinese directly, the poison refers to a female use perfume will of wildness, does not conform to the traditional custom, it cannot be loved by women customers. Translators use "Bai Ai Shen" to translate the word with more feminine and sense of beauty. So "Bai Ai Shen" is recommendable and successful translation.

\section{The Methods OF COSMETIC BRAND TRANSLATION}

In the process of cosmetic brand name translation, it requires the use of language, marketing and aesthetic aspects of the comprehensive knowledge. The methods of translation mainly include transliteration, free translation, combination of transliteration and free translation and conversion. The classification of translation methods is totally equivalence between trademark sounds, shape, and righteousness.

Brand name is simple in structure. However, brand name with informative function, aesthetic function and vocative function, due to the particularity of trademark function, translation also certain complexity, its translation to the appropriate translation theory and method as guidance. Study shows that, among all the translation methods, transliteration accounted for 66 percent, while other translation methods accounted for a smaller proportion. [9]

For translating the domestic cosmetics brand, literal translation is very difficult, and the method of the combination of sound and meaning is more difficult. Although these four kinds of translation methods are objective existence, the function is not completely consistent. Although the transliteration method is simple, it cannot translate the characteristics of trademark, and cannot directly convey the meaning of the brand name. Although literal translation is more complete to the trademark name of meaning expression, but cannot be the condensation, concise function characteristic to express.

The free translation has no corresponding relation in pronunciation and meaning, but it expresses the meaning of positive association, and it is an ideal method of translation. The combination of sound and meaning is the most ideal choice for brand name translation. Because a good translation conveys a certain practical significance, which not only in the form of reproduction beauty of pronunciation of the original name, but also reflects the characteristics of products.

\section{A. Transliteration}

The essence of the transliteration is to keep the original taste, which refers to maintain the pronunciation of original language, and reshape it with new words using target language. Its greatest advantage is to retain the beautiful sound of original trademark, and highlights the exotic feelings of the original. This kind of cosmetic brand name translation can convey the original cultural connotation. The chosen of similar pronunciation, can make cosmetic brand 
feel the meaning of originals, but let cosmetic brand vivid, which can satisfy the customer's consumption psychology.

Transliterations are mostly coined, with little specific meaning in target language. Therefore, transliteration keeps the phonologic beauty of the original trademark. But it does not conform to Chinese rule completely. However, reader can find its ulterior motives in transliteration. The chosen of words are careful and full of attentive. Thus it is novel and unique, to meet the curiosity of female consumers and aesthetic psychology, and relatively straightforward, for translation brought convenience. [10] Many cosmetic trademarks are inclined to adopt this method of translation. For example, "Clean Clear" is translated into "Ke Ling Ke Li” (可伶可俐). The product is faced with young women, which is smart, independent and full of passion. The translation just matches the function of acne cure, and is consistent with the brand positioning. Other typical instances like "Nuskin" translated as “Ru Xin" (如新) conveys the aesthetic characteristics of the brand vividly, lead to a better aesthetic imagination, and stimulate the desire to buy.

\section{B. Free Translation}

In English and Chinese translation, the literal translation and free translation is the translation of two important methods. Transliteration and free translation can be adopted to realize the effective cultural transplantation. Because of the age, sex, and the environment of the consumer, the psychological tendency of shopping is different.

Therefore, trademark designers should make careful investigation and analysis of the product sales market and sales target, the audience's psychological, and then carry out the brand's creativity and positioning. [11] Phonological refers to the trademark pronunciation loud, fast paced, with strong extensibility, conveyed in the formerly known as the theme of information at the same time, to meet the aesthetic psychology of Chinese people, the use of alliteration, rhyme, assonance and onomatopoeia translation methods, so that the translation read catchy sound of loud reading, full of appeal and easy to remember. Such as cosmetics "Max Factor", is translated into "Mi Si Fo Tuo" (蜜丝佛陀). The word "Max" means the largest, "Factor" means the sellers, and it also not an exactly item of transliteration. When the source language and the target language have a large cultural or linguistic structure difference, the method of free translation can be adopted.

Free translation has more freedom and flexibility. It focuses more on the reproduction of the original meaning. Translators need to translate the cosmetic brand based on the full understanding of the original, to express the information contained in the original using target language. Thus they can achieve advertising vocative functions. When there is a big difference between English and Chinese language, transliteration may lead to misunderstanding among Chinese consumers. Like “Elizabeth Arden" (伊丽莎白雅顿) brand translation is just a translation of its name. It is difficult to accept the meaning of the cosmetic at first sight. Then free translation is a good choice, for that Chinese consumer can understand the original communicative intention and thoughts of the cosmetic brand easily.

\section{Conversion}

If transliteration and free translation cannot reproduce the original trademark characteristics vividly, we can set aside the phonology and meaning of original brand, and take unconventional method. That is what we call conversion method. It is not confined to the simple phonological similarity, or its literal meaning. The translator can fully explore new ideas, new, bold innovation, in order to give the readers unlimited reverie. [12] In other words, conversion method is an unconventional special method, refers to be based on the premise of the cultural customs of the target language countries and rename the good using unconventional thoughts.

Although the translation is quite different with the original meaning of the target brand, it can achieve the same effect with above methods. Such as the American brand “Neutrogena", which is translated as “Lu De Qing” (露得清), derives from the Latin word "Neutralis" and "Genus". The meaning of the Latin word is newborn. The combination of the two words implies the meaning of "creating the natural effect". The brand name is famous for a kind of comfortable clean skin soap at its first found time. We translate “Neutrogena” to “Lu De Qing” (露得清), although this translation does not have much literal meaning, we cannot but agree that this translation coincide well with the original intention of clean fresh and clear in its brand story.

\section{Simplification}

Cosmetic brand translation should reflect the information and function as far as possible. If we cannot translate a brand using the above four methods, we can just simplify the brand, and make it short and memorable. Such as "Safeguard" soap is translated to "Shu Fu Jia" (舒肤佳). "Shu” give people a comfortable and smooth feel trademark translation, "Fu" pointed out the role of the production, and "Jia" is implied goods excellent effect. This is a simple but wonderful translation, which is easy to read and suit to the realistic meaning.

The translation of a trademark is almost concise, avoid uncommon word, make full use of the information behind the Chinese words, and stimulate consumer endless imagination, easy to remember. Such as shampoo "Head Shoulders" translated into "Hai Fei Si" (海飞丝), formerly known as literal translation is "head and shoulders" or "Helen cents silk", translation head and shoulders clean let a person reminiscent of beautiful hair like the waves of the sea like scattered on our shoulders, reflects the product characteristics and effectiveness.

Cosmetics translation of the "Fang", "Diane", "Poem" "Blue", "Jade" and other characters, give a person with fresh and fragrant, elegant purity, graceful and beautiful feeling, catering to the majority of women's consumption concept, considering the characteristics of Chinese language, and give a person with visual and auditory beauty to enjoy, and promote the potential customers. 


\section{CONCLUSION}

In summary, there is a certain complexity of the cosmetic brand name translation, but a lot of flexibility is obvious in the way of translating. One brand often cannot be confined to a certain translation. The name is the first information people receive when contacted with cosmetic products. Only brilliant brand name translation can attract customers' eyeballs among all kinds of brands. Therefore, it is necessary to have a rich imagination and artistic inspiration in brand translation. When translate a cosmetic brand name, there are rules of word selection, such as clear, intelligible, innovative and fantastic. We also cannot ignore the lexical characteristics of cosmetic brand translation like extension, association, and feminized diction. There are several principles of cosmetic brand translation.

Translators should not only use the linguistic advantages but also make use of cultural identity. Different kinds of translation methods are mentioned above and different methods can suit to diverse conditions in cosmetic brand translation. In one word, cosmetic brand translation must be close to the readers so as to achieve the merchandise further promotion and advise the purchase to the performance objective.

\section{ACKNOWLEDGMENT}

I extend my sincere gratitude to a number of people who have contributed to this paper. Without their help, this paper would not have been accomplished.

Here my first acknowledgements should go to all those authors whose books I have consulted and borrowed form, only for their previous research on translation and with the knowledge learnt from them can I have the ability to write this paper.

My heart-felt gratitude also goes to my friends and colleagues for their valuable suggestions and professional guidance, they have benefited me a lot, their constant help and kind encouragement have led me to finish this paper.

\section{REFERENCES}

[1] Jiang J. "A study on chinese English translation of domestic cosmetics trademarks from the perspective of translational equivalence theory." Journal of Yichun College, 2011.

[2] Wu Lili. "On cosmetic brand translation from the perspective of $\mathrm{Xu}$ Yuanchong's three beauty principle.” Overseas English, 2013.

[3] Murphy, M. Brand Strategy. New York: PrenticeHall, 1990.

[4] Chen Quanming. "On the translation of imported goods trademarks and methods." Chinese Translators, vol. 6, 2001, pp:11-13.

[5] Zheng Lefeng. "Linguistic features of cosmetic brand names and their translations." Journal of Xichang College, 2010.

[6] An Yaping. "Analysis of Chinese brand names translated by brand names and their translation methods." Shanghai Science and Technology Translation, vol. 4, 2004, pp: 43-46.

[7] Zhu Yan. "Studies on the translation of cosmetic brands." Journal of Tangshan Teachers College, 2014.

[8] Xu Ximing and Xu Yingxin. "Chinese translation of advertising English: aesthetics." Journal of Shangqiu Teachers College, 2006.
[9] Peter. "Approaches to translation." Soins Psychiatrie, vol.2, 1984, pp: 117.

[10] Vermeer, H J. "Skopos and commission in translational action." Readings in Translation Theory Loirnann Kirjapaino Oy, 1989.

[11] Feng Qinghua. Practical Translation Course. Shanghai: Shanghai Foreign Language Education Press, 2005.

[12] Chen Zhendong. "On the translation of English trademarks." Shanghai Translation, vol. 2, 2005, pp: 52-54 